\title{
Relation of Sources of Systemic Fluoride to Prevalence of Dental Fluorosis
}

\author{
Marilyn W. Woolfolk, DDS, MPH* \\ Assistant Professor \\ Barbara Wirth Faja, MPH \\ Research Associate \\ Robert A. Bagramian, DDS, DrPH \\ Professor \\ Department of Prevention and Health Care \\ School of Dentistry \\ University of Michigan \\ Ann Arbor, Ml 48109-1078
}

\begin{abstract}
The prevalence of dental fluorosis in a nonfluoridated area was determined and related to the reported fluoride ingestion histories of the children examined. A convenience sample of 543 schoolchildren in rural areas of Michigan was examined for fluorosis using the Tooth Surface Index of Fluorosis. Questionnaires that asked about previous use of fluorides were sent to parents of all children examined. The response rate was 76 percent (412 usable questionnaires). A criterion for inclusion in the data analysis stipulated that only fluorosed surfaces that occurred bilaterally would be included. Fluorosis was found on 7 percent of all tooth surfaces and only in the mild form. Twenty-two percent of the subjects were classified as having fluorosis. Dietary supplement was the only fluoride that was found to be significantly related to the occurrence of fluorosis. A greater proportion of the subjects with fluorosis listed physicians, rather than dentists, as the source of fluoride prescriptions. The results demonstrate similarities to the fluorosis reported in other studies in nonfluoridated areas, but also suggest the need to minimize the occurrence of fluorosis through proper assessment of a child's fluoride exposure and the judicious use of additional fluoride.
\end{abstract}

Key Words: fluorides, mottled enamel, dental health surveys

Dental fluorosis is a hypocalcification with or without hypoplasia of the dental enamel caused by excessive ingestion of fluoride during the period of tooth calcification and maturation. It has been suggested that fluorosis has become more prevalent among children in this country, in both fluoridated and nonfluoridated areas, as a result of ingestion of fluoride from sources other than drinking water $(1,2)$. Sources included certain in-

Send reprints and correspondence to Dr. Woolfolk. Manuscript received: 6/13/88; returned to author for revision: $8 / 2 / 88$; accepted for publication: $10 / 4 / 88$. fant foods and formulas, fluoride-containing dentifrices, and dietary fluoride supplements.

Dietary fluoride supplements were first accepted by the Council on Dental Therapeutics in 1958. They represent an alternative source of fluoride that can be made available to children who do not have access to optimally fluoridated water supplies. The caries preventive benefits of fluoride supplements have been well documented by research (3-7).

Fluoride supplements are beneficial if patients are maintained on them from birth through age 13 with additional benefits being derived with use until third molars erupt. Current recommendations for providing supplements reflect the desired balance of maximal caries protection with minimal risk of dental fluorosis and stipulate that any dosage regimen should take into consideration the patient's age and the fluoride content of the drinking water (8). Depending upon the circumstances, either the risk of dental fluorosis increases or maximal caries protection is not achieved if these recommendations are not followed conscientiously.

Prescriptions for fluoride supplements can be issued to patients by dentists and physicians, although a national survey conducted by Gift (9) found that more physicians than dentists were prescribing dietary fluorides $(79 \%$ versus $60 \%$ ). State and national surveys from 1975-87 indicate that 32-86 percent of physicians report prescribing supplements (10-13). Investigations into the practices of dentists have found that 21 to 93 percent of surveyed dentists prescribed fluoride supplements $(9,14-16)$. Yet, data also indicate a lack of knowledge among physicians and dentists regarding the use of oral fluoride supplementation in caries prevention and a lack of knowledge of the recommended dosage $(10-11,13,17-19)$.

The present investigation was prompted by concern that recommendations for prescribing fluoride supplements are not being followed appropriately. The authors determined the prevalence of fluorosis in children from a predominantly nonfluoridated area. This article will describe the relation between the reported early use of fluorides and the fluorosis status of the children examined. 


\section{Methods}

A convenience sample of 543 children, aged nine to 13 years, from several rural communities in Michigan, was examined for fluorosis using the Tooth Surface Index of Fluorosis (TSIF) (20). All examinations were performed by one examiner who had been calibrated in the use of the index. To distinguish between fluorosis and nonfluoride opacities, criteria formulated by Russell were used (21). Only permanent teeth were examined. All examinations were conducted in schools with a portable dental chair and a fiber-optic light. A portable computer on site allowed direct data entry by a trained recorder.

\section{"Approximately 37 percent of the children who received fluoride prescriptions from physicians had evidence of fluorosis, compared to only 10 percent who received prescriptions from dentists."}

A questionnaire was developed to obtain information about previous use of fluorides. The instrument was pretested on a sample of 15 colleagues who had children similar in ages to those included in the study. After modifications for clarity, questionnaires were mailed to parents of all children examined for fluorosis. Items on the questionnaire, which included multiplechoice and open-ended questions, asked parents to list all places of residence of their children since birth and whether well water or city water was used. Parents also were asked about use of fluoride supplements, sources of fluoride prescriptions, type of toothpastes used, and infant feeding practices-breast feeding and/or type of formula. To discourage guessing on the part of the respondents, the questionnaires included choices of "I don't know" or "I don't remember" as response options. These answers, indicative of respondents' uncertainty, were then eliminated from the analysis. The fluoride status of all communities listed by respondents was confirmed by consulting the 1980 Fluoridation Census (22) to determine if water fluoridation was present during the children's years of reported residence. Years of reported well water use were considered "no fluoride" years, as the study area is known to have negligible levels of natural fluoride and it was not within the scope of the present investigation to determine fluoride levels in well water for those subjects who may not have been life-long residents of the area.

The TSIF was used to examine individual tooth surfaces for fluorosis; however, a criterion for data analysis of the fluorosed surfaces was added. Because calcification of teeth usually occurs bilaterally, a similar effect of the ingestion of fluoride would normally be found on bilateral teeth. Therefore, only those scores found on bilateral surfaces were included in the data analysis. Data were analyzed and reported on the basis of individual tooth surfaces and on the basis of individual subjects who were stratified into two groups: those with fluorosis and those with no fluorosis. A child was considered to have fluorosis if at least two bilateral teeth, anterior or posterior, had surfaces with evidence of fluorosis. Frequency distributions were constructed. Chi-square and Fisher's exact probability tests were performed to determine if relations existed between the fluorosis status of children and reported exposure to fluorides.

\section{Results}

Of the 543 children examined, 412 fluoride histories were returned, a response rate of 76 percent. The prevalence of fluorosis is shown in Table 1. More than threequarters of schoolchildren had no evidence of fluorosis, as defined previously for individuals. A slightly larger proportion of females than males had fluorosis, 25.2 percent versus 19.1 percent, respectively. However, there was no statistically significant relation between fluorosis status and sex.

The distribution of TSIF scores of all permanent tooth surfaces of the children is shown in Table 2. About 93 percent of the surfaces examined had no fluorosis. Of the approximately 7 percent of the surfaces that had evidence of fluorosis, the majority $(6.1 \%$ of all surfaces) showed only the mildest form of the condition--a score of one.

Table 3 displays the distribution of TSIF scores by age. The youngest children, nine and ten years, displayed the highest proportions of surfaces affected by fluorosis, 11.8 and 8.9 percent, respectively. The largest proportion of surfaces without fluorosis was among the older children. Among those 12 years old, fluorosis was

TABLE 1

Distribution of Schoolchildren by Fluorosis Status, Michigan: 1986

\begin{tabular}{|c|c|c|c|c|c|c|}
\hline & \multicolumn{2}{|c|}{ No Fluorosis } & \multicolumn{2}{|c|}{ Fluorosis } & \multicolumn{2}{|c|}{ Total } \\
\hline & $\%$ & $(n)$ & $\%$ & $(n)$ & $\%$ & $(n)$ \\
\hline Schoolchildren & 77.7 & $(320)$ & 22.3 & $(92)$ & 100.0 & (412) \\
\hline Males & 80.9 & $(157)$ & 19.1 & $(37)$ & 100.0 & (194) \\
\hline Females & 74.8 & $(163)$ & 25.2 & $(55)$ & 100.0 & $(218)$ \\
\hline
\end{tabular}

TABLE 2

Distribution of TSIF Scores for All Permanent Tooth Surfaces of Schoolchildren, Michigan: 1986

\begin{tabular}{lccccc}
\hline & \multicolumn{5}{c}{ TSIF Score } \\
\cline { 2 - 6 } & 0 & 1 & 2 & 3 & 4 \\
\hline Percent & 93.1 & 6.1 & 0.6 & 0.2 & 0.0 \\
No. of surfaces & 16,028 & 1,044 & 107 & 26 & 3 \\
\hline
\end{tabular}


TABLE 3

Percent Distribution of TSIF Scores for All Permanent Tooth Surfaces by Age of Schoolchildren, Michigan: 1986

\begin{tabular}{|c|c|c|c|c|c|c|}
\hline \multirow{2}{*}{$\begin{array}{l}\text { Age } \\
\text { (Years) }\end{array}$} & \multirow{2}{*}{$\begin{array}{c}\text { No. of } \\
\text { Surfaces }\end{array}$} & \multicolumn{5}{|c|}{ TSIF Score } \\
\hline & & 0 & 1 & 2 & 3 & 4 \\
\hline 9 & 2,854 & 88.2 & 10.7 & 1.0 & 0.1 & 0.0 \\
\hline 10 & 6,050 & 91.1 & 7.5 & 1.0 & 0.4 & 0.0 \\
\hline 11 & 5,892 & 95.3 & 4.3 & 0.3 & 0.0 & - \\
\hline 12 & 1,868 & 98.3 & 1.7 & - & - & - \\
\hline 13 & 544 & 100.0 & 一 & - & - & - \\
\hline
\end{tabular}

TABLE 4

Distribution of Schoolchildren by Reported Exposure to Fluoride Water Supply and Fluorosis Status, Michigan: 1986

\begin{tabular}{lcccc}
\hline \multirow{2}{*}{$\begin{array}{l}\text { Duration of } \\
\text { Fluoride Exposure }\end{array}$} & \multicolumn{2}{c}{ No Fluorosis } & & \multicolumn{2}{c}{ Fluorosis } \\
\cline { 2 - 3 } \cline { 5 - 5 } & $\%$ & $(n)$ & & $(n)$ \\
\hline 0 years & 78.6 & $(191)$ & 21.4 & $(52)$ \\
1-4 years & 74.4 & $(67)$ & 25.6 & $(23)$ \\
5-8 years & 78.5 & $(67)$ & 21.5 & $(17)$ \\
\hline
\end{tabular}

found on 1.7 percent of the surfaces. No fluorosis was found among the 13-year-old children.

Parents were asked to list all places of residence for their children, but only places of residence in the first eight years of each child's life were included for analysis. For most children, calcification of the enamel of all teeth (except third molars) would have occurred during that time span. The distribution of children by previous exposure to fluoridated community water supplies and fluorosis status is found in Table 4. Years of exposure were combined to form three categories: no fluoridated water exposure, one to four years, and five to eight years. Although slightly more fluorosis was found among those children whose cumulative exposure time was one to four years, no striking patterns or statistically significant relations were found.

Table 5 shows the distribution of children by fluorosis status in relation to the reported frequency of taking dietary fluoride supplements: "regularly as prescribed," "not on a regular basis," or "never." Significant relations were found between fluorosis status and reported use of fluoride supplements (chi square, $P<$ .01). Regular users of supplements were more likely to show evidence of fluorosis, 32.4 percent versus only 13.4 percent of those who never took supplements. Those who reported never taking fluoride supplements were more likely not to have fluorosis. Approximately 87 percent of nonusers were fluorosis-free; 67.6 percent of regular users had no fluorosis. Of the other sources of fluoride investigated, toothpaste and infant formulas, none were found to be related to fluorosis.
TABLE 5

Distribution of Schoolchildren by Reported Use of Dietary Fluoride Supplements and Fluorosis Status, Michigan: 1986

\begin{tabular}{lrrrr}
\hline & \multicolumn{2}{c}{ No Fluorosis } & \multicolumn{2}{c}{ Fluorosis } \\
\cline { 2 - 3 } \cline { 5 - 6 } Use & $\%$ & $(n)$ & $\%$ & $(n)$ \\
\hline Regular & 67.6 & $(115)$ & 32.4 & $(55)$ \\
Less than regular & 78.9 & $(71)$ & 21.1 & $(19)$ \\
Never & 86.5 & $(103)$ & 13.4 & $(16)$ \\
\hline
\end{tabular}

TABLE 6

Distribution of Schoolchildren by Source of Dietary Fluoride Supplement Prescription and Fluorosis Status, Michigan: 1986

\begin{tabular}{lccrrr}
\hline & \multicolumn{2}{c}{ No Fluorosis } & & \multicolumn{2}{c}{ Fluorosis } \\
\cline { 2 - 3 } \cline { 5 - 6 } Source & $\%$ & $(n)$ & & $\%$ & $(n)$ \\
\hline Physician & 63.4 & $(102)$ & & 36.6 & $(59)$ \\
Dentist & 89.7 & $(70)$ & & 10.3 & $(8)$ \\
\hline
\end{tabular}

Parents were asked to identify who prescribed fluoride supplements for their children. Of those who reported taking supplements, 67 percent reported receiving the fluoride prescriptions from physicians; 33 percent received them from dentists. The distribution of fluorosis among the children as related to the reported source of prescription is found in Table 6. Statistically significant relations were found between the presence of fluorosis and source of fluoride supplement prescriptions (Fisher's exact test, $P<.01$ ). Approximately 37 percent of the children who received fluoride prescriptions from physicians had evidence of fluorosis, compared to only 10 percent who received prescriptions from dentists.

\section{Discussion}

Although examiner variability and the use of different populations and indices prevent direct comparisons, the prevalence of fluorosis found in the present study is similar to that reported previously in other areas of negligible fluoride in the water. Oldak and Leverett (23), using Dean's index, reported that 22 percent of first and second grade children living in a nonfluoridated area of New York experienced dental fluorosis in the permanent teeth. Using the TSIF, Driscoll et al. (24) found a similar percentage of fluorosis-free surfaces $(94.1 \%)$ on subjects eight to 16 years of age in studies of rural communities with negligible levels of fluoride in the water supply.

Despite the relatively low prevalence and mild form of fluorosis found, the question arises as to which source of fluoride might increase the risk of fluorosis, especially in areas where fluoridated water is not expected to be a factor. The fluoride histories of the subjects in this study provide some insight, although some 
concerns should be noted. Information from the surveys is based on self-reported data and depends to a great extent on the parents' ability to recall experiences specifically for each of their children. Recognizing that caution must be observed when interpreting self-reported data, however, the histories provided the following information.

Various exposures to community fluoridated water supplies were reported. Lack of specific knowledge of well water fluoride levels could result in an underestimate of fluoride exposure. It was found, however, that length of reported exposure, ranging from none to eight years of exposure during tooth calcification, was not related to the occurrence of fluorosis. Other sources of possible fluoride exposure, such as use of fluoride toothpastes or ingesting certain types of infant formula, were not found to be related to the occurrence of fluorosis, either. Dietary supplements were the only vehicle of fluoride that was significantly related to the fluorosis found among the population in the present study. Regular users, defined as taking supplements "regularly as prescribed," were more likely to show evidence of fluorosis than those who took them infrequently or not at all. This relation is similar to that reported in other studies that found a greater proportion of fluorosis among children who took fluoride supplements $(4,7,25)$. Like other studies, however, the fluorosis observed was mild, which is considered "acceptable" by some researchers $(7,25)$.

\section{"Dietary supplements were the only vehicle of fluoride that was significantly related to the fluorosis found among the population in the present study."}

The fluorosis found in this study was more likely to be observed in children who reportedly received supplement prescriptions from physicians rather than dentists. Since two-thirds of the fluoride supplement prescriptions were reportedly issued by physicians, it appears that many physicians are assuming a responsibility for preventive dental care. This trend is one that public health advocates support and welcome. The fact that 64 percent of children under the age of five have never visited a dentist (26) indicates the need for other health care providers to be involved in preventive oral health care of young children.

In light of the findings of this study as well as others, there is concern, however, that proper protocol regarding fluoride supplements is still not being followed. Horowitz (27) indicates that the complexity of the dosage schedule is one of the barriers to the appropriate use of fluoride supplements, as is a lack of emphasis placed on the appropriate use of fluorides by faculty teaching in undergraduate and graduate schools.
Further complicating the issue is the fact that, until 1979, the dosage schedule for fluoride supplements as recommended by the American Dental Association was different from that of the American Academy of Pediatrics (28). Changing guidelines for supplement prescriptions may have gone unnoticed or were ignored. A study of physicians by Margolis and others (12) found positive changes in attitudes and practices following an educational program about fluoride supplements. However, in a study that evaluated the fluoride prescribing practices of medical residents, Pinkerton et al. (29) found that, even when new information was learned, correspondingly appropriate prescribing behavior did not necessarily follow. Also, while physicians have indicated that, among the criteria considered when prescribing supplements, the amount of fluoride in the drinking water is a major criterion (9), a relatively low percentage know the fluoride content of the water (11) or actually assay the water to determine the fluoride content $(13,18)$. It appears, then, that while many physicians recognize the importance of fluoride supplements and can cite the correct guidelines for their use, recommendations are not being followed either by the physicians or parents and children, or both.

Results of the present investigation suggest that fluoride supplements contribute to the prevalence of fluorosis and more likely may be prescribed inappropriately by physicians. Although the amount of fluorosis that is being reported may be more of an esthetic than health concern, its unnecessary occurrence can and should be purposefully minimized through proper assessment of the child's fluoride exposure and the judicious use of additional fluoride.

\section{References}

1. Leverett $\mathrm{DH}$. Fluoride and the changing prevalence of dental caries. Science 1982 Jul;217;26-30.

2. Leverett DH. Prevalence of dental fluorosis in fluoridated and nonfluoridated communities-a preliminary investigation. J Public Health Dent 1986 Fall;46:184-7.

3. Forrester DJ, Schulz EM, eds. International workshop on fluorides and dental caries reductions. Baltimore: University of Maryland, 1974:105-6.

4. Aasenden R, Peebles TC. Effects of fluoride supplementation from birth on human deciduous and permanent teeth. Arch Oral Biol 1974 Apr;19:321-6.

5. Margolis F], Reames HR, Freshman E, Macauley JC, Mehaffey H. Fluoride: ten-year prospective study of deciduous and permanent dentition. Am I Dis Child 1975;129:794-800.

6. Aasenden R, Peebles TC. Effects of fluoride supplementation from birth on dental caries and fluorosis in teenaged children. Arch Oral Biol 1978;23:111-5.

7. Hennon DK, Stookey GK, Beiswanger BB. Fluoride-vitamin supplements: effects on dental caries and fluorosis when used in areas with suboptimum fluoride in the water supply. J Am Dent Assoc 1977 Nov;95:965-71.

8. Council on Dental Therapeutics. Prescribing fluoride supplements. In: American Dental Association. Accepted dental therapeutics, 40th ed. Chicago: ADA, 1984:395-420.

9. Gift HC, Milton BB, Walsh V. The role of the health professional in the delivery of caries prevention. Chicago: American Dental Association Health Foundation, 1983.

10. Margolis FJ, Burt BA, Schork MA, Bashshur RL, Whittaker BA, Burns TL. Fluoride supplements for children. A survey of physicians' prescription practices. Am J Dis Child 1980 Sept;134:865-8.

11. Siegel C, Gutgesell ME. Fluoride supplementation in Harris County, Texas. Am J Dis Child 1982 Jan;136:61-3. 
12. Margolis FJ, Chesney BK, Schork A. Fluoride supplements. Changes in physicians' attitudes and practices following an intensive, multifaceted educational program. Am J Dis Child 1987 Jan;141:72-6.

13. Kuthy RA, McTigue DJ. Fluoride prescription practices of Ohio physicians. J Public Health Dent 1987 Fall;47:172-6.

14. Bureau of Economic Research and Statistics. Prescribing and dispensing habits of dentists, 1975. Chicago: American Dental Association, 1976.

15. Levy SM. Expansion of the proper use of systemic fluoride supplements. J Am Dent Assoc 1986 Jan;112:30-4.

16. Levy SM, Rozier RG, Bawden JW. Use of systemic fluoride supplements by North Carolina dentists. J Am Dent Assoc 1987 Mar; 114:347-50.

17. Messimer S, Hickner J. Oral fluoride supplementation: improving practitioner compliance by using a protocol. J Fam Pract 1983 Nov; 17:821-5.

18. Levy SM, Bawden JW, Bowden BS, Rozier GR. Fluoride analyses of patient water supplies requested by North Carolina health professionals. Am J Public Health 1984 Dec;74:1412-4.

19. Rigilano JC, Freidler EM, Ehemann LJ. Fluoride prescribing patterns among primary care physicians. I Fam Pract 1985;21:381-5.

20. Horowitz HS, Driscoll WS, Meyers RJ, Heifetz SB, Kingman A. A new method for assessing the prevalence of dental fluorosis-the tooth surface index of fluorosis. J Am Dent Assoc 1984 Jul;109:3741.

21. Russell AL. The differential diagnosis of fluoride and nonfluoride enamel opacities. J Public Health Dent 1961;21:143-6.
22. Centers for Disease Control. Fluoridation census 1980. Atlanta: Public Health Service, DHHS, 1984.

23. Oldak SB, Leverett DH. Dental fluorosis in a non-fluoridated community [Abstract]. J Dent Res 1984 Mar;63(Spec Iss):197.

24. Driscoll WS, Horowitz HS, Meyers RJ, Heifetz SB, Kingman A Zimmerman ER. Prevalence of dental caries and dental fluorosis in areas with negligible, optimal, and above-optimal water fluoride concentrations in drinking water. J Am Dent Assoc 1986 Jul;113:29-33.

25. Powell JA, Norbert LD, Hargreaves JA. Fluorosis and dental caries in children receiving fluoride supplementation. [Abstract]. J Dent Res 1983 Mar;62:203.

26. Jack SS. Use of dental services: United States, 1983 (Advanced Data). Hyattsville, MD: National Center for Health Statistics, 1986; DHHS pub no (PHS) 86-1250 (Vital and health statistics; no 122.)

27. Horowitz AM. Ways to improve/increase appropriate use of dietary fluorides. J Dent Child 1985 Jul-Aug;52:269-74.

28. American Academy of Pediatrics, Committee on Nutrition. Fluoride supplementation: revised dosage schedule. Pediatrics 1979 Jan;63:150-2.

29. Pinkterton RE, Tiananoff N, Williams JL, Tapp JT. Resident physician performance in a continuing education format. J Am Med Assoc 1980 Nov 14;244:2183-5.

30. Levy SM. Systemic fluoride supplementation in an academic family practice setting. J Fam Pract 1987;24:532-6. 